\title{
Erratum to: Effective dose to adult patients from 338 radiopharmaceuticals estimated using ICRP biokinetic data, ICRP/ICRU computational reference phantoms and ICRP 2007 tissue weighting factors
}

Martin Andersson ${ }^{1,2}$

\section{Correspondence:}

martin.andersson@med.lu.se

'Medical Radiation Physics, Department of Clinical Sciences Malmö, Lund University, Skåne University Hospital, Malmö, Sweden ${ }^{2}$ Department of Radiation Sciences, Umeå University, Umeå, Sweden

\section{Correction of the article}

In the results section of the abstract the two sentences

1. For $79 \%$ of the radiopharmaceuticals, the new calculations gave a lower effective dose per unit administered activity than earlier estimated." Should be "For $63 \%$ of the radiopharmaceuticals, the new calculations gave a lower effective dose per unit administered activity than earlier estimated."

2. "As a mean for all radiopharmaceuticals, the effective dose was $25 \%$ lower." Should be"As a mean for all radiopharmaceuticals, the effective dose was 11 \% lower."

In the results section in the main article four sentences should be changed

1. "The calculated values are lower than earlier presented values for $79 \%$ of the radiopharmaceuticals." Should be "The calculated values are lower than earlier presented values for $63 \%$ of the radiopharmaceuticals."

2. "As a mean for all 338 radiopharmaceuticals, the values are $25 \%$ lower." Should be "As a mean for all 338 radiopharmaceuticals, the values are $11 \%$ lower."

3. "The effective doses are larger for females than for males in $62 \%$ of all 338 radiopharmaceuticals." Should be "The effective doses are larger for females than for males in $99 \%$ of all 338 radiopharmaceuticals."

4. "Only for ${ }^{125}$ I Iodine Hippuran with unilateral renal blockage and an abnormal kidney function there is a difference of more than $100 \%$ between the new and the old E/ $\mathrm{A}_{0}$ values." Should be "Only for ${ }^{99 \mathrm{~m}} \mathrm{Tc}$ Apcitide and ${ }^{99 \mathrm{~m}} \mathrm{Tc}$ labelled colloids, small colloids and normal liver condition there is a difference of more than $100 \%$ between the new and the old $\mathrm{E} / \mathrm{A}_{0}$ values."

\section{Springer}

(C) 2015 Andersson. Open Access This article is distributed under the terms of the Creative Commons Attribution 4.0 International License(http://creativecommons.org/licenses/by/4.0/), which permits unrestricted use, distribution, and reproduction in any medium, provided you give appropriate credit to the original author(s) and the source, provide a link to the Creative Commons license, and indicate if changes were made. 
In the Discussion section in the main article eight sentences should be changed

1. "For radiopharmaceuticals with a significant uptake in adipose tissue as for ${ }^{14} \mathrm{C}$ - and ${ }^{3} \mathrm{H}$-labelled neutral fat and free fatty acids or in the male gonads, the effective dose will be higher for males than for females." Should be "For radiopharmaceuticals with a significant uptake in adipose tissue as for ${ }^{14} \mathrm{C}$ - and ${ }^{3} \mathrm{H}$-labelled neutral fat and free fatty acids, the effective dose will be higher for males than for females."

2. "For ${ }^{18}$ F-labelled substances, $\mathrm{E} / \mathrm{A}_{0}$ varies between 0.013 and $0.019 \mathrm{mSv} / \mathrm{MBq}$ (less than a factor of 1.5)." Should be "For ${ }^{18}$ F-labelled substances, $\mathrm{E} / \mathrm{A}_{0}$ varies between 0.013 and $0.021 \mathrm{mSv} / \mathrm{MBq}$ (a factor of 1.6)."

3. "For ${ }^{11} \mathrm{C}$-substances, $\mathrm{E} / \mathrm{A}_{0}$ varies between 0.0025 and $0.0055 \mathrm{mSv} / \mathrm{MBq}$ (around a factor of 2.2)." Should be "For ${ }^{11} \mathrm{C}$-substances, $\mathrm{E} / \mathrm{A}_{0}$ varies between 0.0011 and $0.0087 \mathrm{mSv} / \mathrm{MBq}$ (around a factor of 8.0). "

4. "Also for ${ }^{99 \mathrm{~m}} \mathrm{Tc}$-labelled substances, the range of $\mathrm{E} / \mathrm{A}_{0}$ values is limited to 0.0017 to $0.016 \mathrm{mSv} / \mathrm{MBq}$ (a factor of 9.6)." Should be "Also for ${ }^{99 \mathrm{~m}}$ Tc-labelled substances, the range of $\mathrm{E} / \mathrm{A}_{0}$ values is limited to 0.0022 to $0.020 \mathrm{mSv} / \mathrm{MBq}$ (a factor of 8.8)."

5. "For all the ${ }^{18} \mathrm{~F}$ substances, there is a reduction in effective dose estimation by $29 \%$ in average." Should be "For all the ${ }^{18} \mathrm{~F}$ substances, there is a reduction in effective dose estimation by $26 \%$ in average."

6. "For ${ }^{11} \mathrm{C}$-substances, two radiopharmaceuticals show a higher effective dose and 11 have a lower effective dose than previously published values." Should be "For ${ }^{11} \mathrm{C}$-substances, nine radiopharmaceuticals show a higher effective dose and four have a lower effective dose than previously published values."

7. "In 50 of the $62^{99 \mathrm{~m}} \mathrm{Tc}$-substances, the effective dose estimations give lower values than previous estimations." Should be "In 38 of the $62{ }^{99 \mathrm{~m}} \mathrm{Tc}$-substances, the effective dose estimations give lower values than previous estimations."

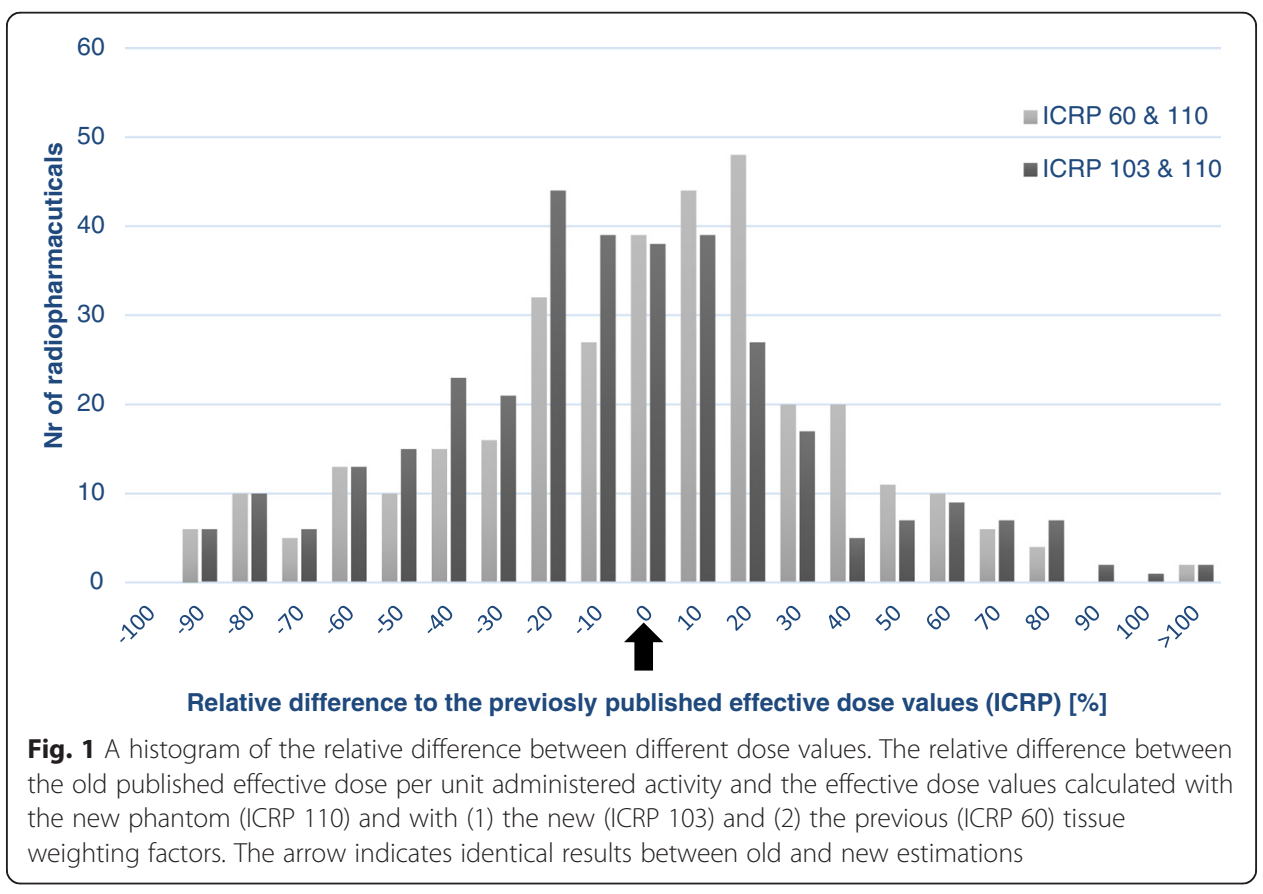


Table 1 Effective dose from the 55 radiopharmaceuticals in ICRP publication 106, determined using three different methods

\begin{tabular}{|c|c|c|c|c|c|c|c|}
\hline Radiopharmaceuticals & $\left(\mathrm{E} / \mathrm{A}_{0}\right) 1[\mathrm{mSv} / \mathrm{MBq}]$ & $\left(E / A_{0}\right) 2[\mathrm{mSv} / \mathrm{MBq}]$ & $\begin{array}{l}\left(\left(E / A_{0}\right) 2-\left(E / A_{0}\right) 1\right) / \\
\left(E / A_{0}\right) 1[\%]\end{array}$ & $\begin{array}{l}\left(E / A_{0}\right) 3 \\
{[\mathrm{mSV} / \mathrm{MBq}]}\end{array}$ & $\begin{array}{l}\left(\left(E / A_{0}\right) 3-\left(E / A_{0}\right) 1\right) / \\
\left(E / A_{0}\right) 1[\%]\end{array}$ & $\begin{array}{l}\left(E / A_{0}\right) 3 \text { male } \\
{[\mathrm{mSV} / \mathrm{MBq}]}\end{array}$ & $\begin{array}{l}\left(E / A_{0}\right) 3 \text { female } \\
{[\mathrm{mSV} / \mathrm{MBq}]}\end{array}$ \\
\hline Phantom & MIRD & ICRP/ICRU & & ICRP/ICRU & & ICRP/ICRU & ICRP/ICRU \\
\hline $\mathrm{W}_{\mathrm{T}}$ & ICRP 60 & ICRP 60 & & ICRP 103 & & ICRP 103 & ICRP 103 \\
\hline $\begin{array}{l}{ }^{3} \mathrm{H} \text { Tritium labelled neutral fat \& free fatty } \\
\text { acids }\end{array}$ & $2.2 \mathrm{E}-01$ & $9.81 \mathrm{E}-02$ & -55 & $1.80 \mathrm{E}-01$ & -18 & 2.44E-01 & $1.16 \mathrm{E}-01$ \\
\hline${ }^{11} \mathrm{C}$ Carbon acetate & $3.5 \mathrm{E}-03$ & $4.26 \mathrm{E}-03$ & 22 & 3.65E-03 & 4 & 3.33E-03 & 3.97E-03 \\
\hline${ }^{11} \mathrm{C}$ Carbon amino acids & $5.6 \mathrm{E}-03$ & $5.76 \mathrm{E}-03$ & 3 & $5.26 \mathrm{E}-03$ & -6 & $4.91 \mathrm{E}-03$ & $5.61 \mathrm{E}-03$ \\
\hline${ }^{11} \mathrm{C}$ Carbon brain receptor substances & $4.3 \mathrm{E}-03$ & $3.70 \mathrm{E}-03$ & -14 & $3.62 \mathrm{E}-03$ & -16 & $3.23 \mathrm{E}-03$ & 4.00E-03 \\
\hline${ }^{11} \mathrm{C}$ Carbon methionine & $8.4 \mathrm{E}-03$ & $5.88 \mathrm{E}-03$ & -30 & 5.11E-03 & -39 & 4.50E-03 & $5.72 \mathrm{E}-03$ \\
\hline${ }^{11} \mathrm{C}$ Carbon $\left(2-{ }^{11} \mathrm{C}\right)$ thymidine & 2.7E-03 & $3.01 \mathrm{E}-03$ & 11 & $3.04 \mathrm{E}-03$ & 13 & 2.77E-03 & $3.32 \mathrm{E}-03$ \\
\hline${ }^{11} \mathrm{C}$ Carbon, realistic maximum & $1.1 \mathrm{E}-02$ & $6.04 \mathrm{E}-03$ & -45 & $5.08 \mathrm{E}-03$ & -54 & 4.36E-03 & $5.80 \mathrm{E}-03$ \\
\hline $\begin{array}{l}{ }^{14} \mathrm{C} \text { Carbon labelled neutral fat \& free fatty } \\
\text { acids }\end{array}$ & $2.1 E+00$ & $1.88 \mathrm{E}+00$ & -10 & $2.65 E+00$ & 26 & $3.14 \mathrm{E}+00$ & $2.15 E+00$ \\
\hline $\begin{array}{l}{ }^{14} \mathrm{C} \text { Carbon labelled urea, normal case, orally } \\
\text { administered }\end{array}$ & $3.1 \mathrm{E}-02$ & 2.67E-02 & -14 & $2.72 \mathrm{E}-02$ & -12 & $2.46 \mathrm{E}-02$ & $2.98 \mathrm{E}-02$ \\
\hline${ }^{15} \mathrm{O}$ Oxygen water & $1.1 \mathrm{E}-03$ & $9.63 \mathrm{E}-04$ & -12 & 9.33E-04 & -15 & $8.72 \mathrm{E}-04$ & $9.93 \mathrm{E}-04$ \\
\hline${ }^{18} \mathrm{~F}$ Fluoride labelled amino acids & $2.3 \mathrm{E}-02$ & $2.27 \mathrm{E}-02$ & -1 & 2.07E-02 & -10 & $1.92 \mathrm{E}-02$ & $2.21 \mathrm{E}-02$ \\
\hline $\begin{array}{l}{ }^{18} \mathrm{~F} \text { Fluoride labelled brain receptor } \\
\text { substances }\end{array}$ & $2.8 \mathrm{E}-02$ & 2.01E-02 & -28 & $2.02 \mathrm{E}-02$ & -28 & $1.82 \mathrm{E}-02$ & $2.22 \mathrm{E}-02$ \\
\hline${ }^{18}$ F Fluoride FDG & $1.9 \mathrm{E}-02$ & $1.69 \mathrm{E}-02$ & -11 & $1.71 \mathrm{E}-02$ & -10 & $1.53 \mathrm{E}-02$ & $1.88 \mathrm{E}-02$ \\
\hline${ }^{18} \mathrm{~F}$ Fluoride L-dopa & $2.5 \mathrm{E}-02$ & $1.75 \mathrm{E}-02$ & -30 & $1.57 \mathrm{E}-02$ & -37 & $1.37 \mathrm{E}-02$ & $1.76 \mathrm{E}-02$ \\
\hline${ }^{51} \mathrm{Cr}$ Chromium EDTA & $2.0 \mathrm{E}-03$ & $1.65 \mathrm{E}-03$ & -18 & $1.43 \mathrm{E}-03$ & -29 & $1.23 \mathrm{E}-03$ & $1.62 \mathrm{E}-03$ \\
\hline${ }^{67} \mathrm{Ga}$ Gallium citrate & $1.0 \mathrm{E}-01$ & $9.29 \mathrm{E}-02$ & -7 & $9.08 \mathrm{E}-02$ & -9 & $8.14 \mathrm{E}-02$ & $1.00 \mathrm{E}-01$ \\
\hline${ }^{68}$ Ga Gallium labelled EDTA & $4.0 \mathrm{E}-02$ & $2.41 \mathrm{E}-02$ & -40 & $2.14 \mathrm{E}-02$ & -47 & 1.89E-02 & 2.40E-02 \\
\hline${ }^{75}$ Se Selenium labelled amino acids & $2.2 \mathrm{E}+00$ & $2.39 \mathrm{E}+00$ & 8 & $2.27 \mathrm{E}+00$ & 3 & $2.14 \mathrm{E}+00$ & $2.39 \mathrm{E}+00$ \\
\hline
\end{tabular}


Table 1 Effective dose from the 55 radiopharmaceuticals in ICRP publication 106, determined using three different methods (Continued)

\begin{tabular}{|c|c|c|c|c|c|c|c|}
\hline${ }^{75}$ Se Selenium labelled bile acid SeHCAT & $6.9 \mathrm{E}-01$ & 3.01E-01 & -56 & 3.48E-01 & -50 & $3.16 \mathrm{E}-01$ & 3.79E-01 \\
\hline${ }^{99 \mathrm{~m}} \mathrm{Tc}$ Technetium apcitide & 4.7E-03 & $1.13 \mathrm{E}-02$ & 140 & 1.19E-02 & 153 & 1.10E-02 & $1.29 \mathrm{E}-02$ \\
\hline $\begin{array}{l}{ }^{99 \mathrm{~m}} \mathrm{Tc} \text { Technetium labelled small colloids, } \\
\text { intratumoural adm. time to removal } 18 \mathrm{~h}\end{array}$ & 2.0E-03 & $3.14 \mathrm{E}-03$ & 57 & 3.96E-03 & 98 & 3.49E-03 & 4.43E-03 \\
\hline $\begin{array}{l}{ }^{99 \mathrm{~m}} \mathrm{Tc} \text { Technetium labelled small colloids, } \\
\text { intratumoural adm time to removal } 6 \mathrm{~h}\end{array}$ & $1.2 \mathrm{E}-03$ & $1.78 \mathrm{E}-03$ & 48 & 2.24E-03 & 86 & $1.98 \mathrm{E}-03$ & $2.50 \mathrm{E}-03$ \\
\hline${ }^{99 m} \mathrm{Tc}$ Technetium EC, normal renal function & $6.3 \mathrm{E}-03$ & 4.63E-03 & -27 & 3.66E-03 & -42 & 3.04E-03 & 4.29E-03 \\
\hline${ }^{99 m} \mathrm{Tc}$ Technetium ECD & 7.7E-03 & 5.97E-03 & -23 & $5.64 \mathrm{E}-03$ & -27 & $5.01 \mathrm{E}-03$ & $6.27 \mathrm{E}-03$ \\
\hline${ }^{99 m} \mathrm{Tc}$ Technetium furifosmin, exercise & $8.9 \mathrm{E}-03$ & $6.57 \mathrm{E}-03$ & -26 & $6.78 \mathrm{E}-03$ & -24 & $6.16 \mathrm{E}-03$ & 7.40E-03 \\
\hline${ }^{99 m} \mathrm{Tc}$ Technetium furifosmin, resting subject & $1.0 \mathrm{E}-02$ & $6.99 \mathrm{E}-03$ & -30 & 7.19E-03 & -28 & $6.53 \mathrm{E}-03$ & 7.85E-03 \\
\hline${ }^{{ }^{99 m}}$ Tc Technetium labelled HIG & 7.0E-03 & $9.83 \mathrm{E}-03$ & 40 & $9.42 \mathrm{E}-03$ & 35 & 8.93E-03 & $9.92 \mathrm{E}-03$ \\
\hline${ }^{99 \mathrm{~m}} \mathrm{Tc}$ Technetium labelled HM-PAO & $9.3 \mathrm{E}-03$ & $1.05 \mathrm{E}-02$ & 13 & $9.78 \mathrm{E}-03$ & 5 & 8.95E-03 & $1.06 \mathrm{E}-02$ \\
\hline $\begin{array}{l}\text { Tc-99 m Technetium labelled IDA derivatives, } \\
\text { normal hepato-biliary conditions }\end{array}$ & 1.7E-02 & $9.39 \mathrm{E}-03$ & -45 & $9.73 \mathrm{E}-03$ & -43 & 8.93E-03 & $1.05 \mathrm{E}-02$ \\
\hline${ }^{99 \mathrm{~m}} \mathrm{Tc}$ Technetium labelled MAA & $1.1 \mathrm{E}-02$ & $1.34 \mathrm{E}-02$ & 22 & 1.40E-02 & 27 & $1.27 \mathrm{E}-02$ & $1.53 \mathrm{E}-02$ \\
\hline $\begin{array}{l}{ }^{99 m} \mathrm{Tc} \text { Technetium labelled MAG3, normal } \\
\text { renal function }\end{array}$ & 7.0E-03 & $5.12 \mathrm{E}-03$ & -27 & 4.00E-03 & -43 & $3.29 \mathrm{E}-03$ & 4.70E-03 \\
\hline $\begin{array}{l}{ }^{99 m} \mathrm{~m} \text { T Technetium labelled non-absorbable } \\
\text { markers, orally administered fluids }\end{array}$ & $1.9 \mathrm{E}-02$ & $1.06 \mathrm{E}-02$ & -44 & $1.07 E-02$ & -44 & 9.93E-03 & 1.14E-02 \\
\hline $\begin{array}{l}{ }^{99 \mathrm{~m}} \mathrm{Tc} \text { Technetium labelled non-absorbable } \\
\text { markers, orally administered solids }\end{array}$ & $2.4 \mathrm{E}-02$ & $1.13 \mathrm{E}-02$ & -53 & 1.15E-02 & -52 & 1.07E-02 & $1.24 \mathrm{E}-02$ \\
\hline${ }^{99 \mathrm{~m}} \mathrm{Tc}$ Technetium labelled MIBI, exercise & 7.9E-03 & $6.55 \mathrm{E}-03$ & -17 & $6.29 \mathrm{E}-03$ & -20 & $5.80 \mathrm{E}-03$ & $6.78 \mathrm{E}-03$ \\
\hline $\begin{array}{l}{ }^{99 \mathrm{~m}} \mathrm{Tc} \text { Technetium labelled MIBI, resting } \\
\text { subject }\end{array}$ & $9.0 \mathrm{E}-03$ & $6.81 \mathrm{E}-03$ & -24 & $6.61 \mathrm{E}-03$ & -27 & $6.14 \mathrm{E}-03$ & 7.07E-03 \\
\hline${ }^{99 m} \mathrm{Tc}$ Technetium labelled monoclonal & $1.2 \mathrm{E}-02$ & 1.17E-02 & -3 & $1.08 \mathrm{E}-02$ & -10 & 9.95E-03 & 1.16E-02 \\
\hline
\end{tabular}


Table 1 Effective dose from the 55 radiopharmaceuticals in ICRP publication 106, determined using three different methods (Continued)

\begin{tabular}{|c|c|c|c|c|c|c|c|}
\hline${ }^{99 m} \mathrm{Tc}$ Technetium pertechnegas & $1.2 \mathrm{E}-02$ & $1.52 \mathrm{E}-02$ & 26 & $1.50 \mathrm{E}-02$ & 25 & $1.39 \mathrm{E}-02$ & $1.61 \mathrm{E}-02$ \\
\hline $\begin{array}{l}{ }^{99 \mathrm{~m}} \mathrm{Tc} \text { Technetium pertechnetate, intravenous } \\
\text { blocking agent given }\end{array}$ & $4.2 \mathrm{E}-03$ & 4.34E-03 & 3 & $4.02 \mathrm{E}-03$ & -4 & $3.58 \mathrm{E}-03$ & 4.46E-03 \\
\hline $\begin{array}{l}{ }^{99 \mathrm{~m}} \mathrm{Tc} \text { Technetium pertechnetate, intravenous } \\
\text { no blocking agent given }\end{array}$ & $1.3 \mathrm{E}-02$ & $1.60 \mathrm{E}-02$ & 23 & $1.58 \mathrm{E}-02$ & 22 & 1.48E-02 & $1.68 \mathrm{E}-02$ \\
\hline $\begin{array}{l}{ }^{99 m} \mathrm{Tc} \text { Technetium pertechnetate orally, no } \\
\text { blocking agent }\end{array}$ & $1.4 \mathrm{E}-02$ & $6.48 \mathrm{E}-03$ & -54 & $6.36 \mathrm{E}-03$ & -55 & $5.83 \mathrm{E}-03$ & $6.89 \mathrm{E}-03$ \\
\hline $\begin{array}{l}{ }^{99 m} \text { Tc Technetium labelled phosphates and } \\
\text { phosphonates, normal uptake and excretion }\end{array}$ & 5.7E-03 & 4.55E-03 & -20 & $3.99 \mathrm{E}-03$ & -30 & $3.38 \mathrm{E}-03$ & 4.59E-03 \\
\hline${ }^{99 \mathrm{~m}} \mathrm{Tc}$ Technetium labelled erythrocytes & $7.0 \mathrm{E}-03$ & $1.06 \mathrm{E}-02$ & 51 & $1.11 \mathrm{E}-02$ & 59 & $1.02 \mathrm{E}-02$ & $1.20 \mathrm{E}-02$ \\
\hline${ }^{99 \mathrm{~m}} \mathrm{Tc}$ Technetium technegas & $1.5 \mathrm{E}-02$ & 1.79E-02 & 19 & 1.90E-02 & 27 & $1.71 \mathrm{E}-02$ & $2.08 \mathrm{E}-02$ \\
\hline${ }^{99 \mathrm{~m}} \mathrm{Tc}$ Technetium tetrofosmin, exercise & $6.9 \mathrm{E}-03$ & $5.54 \mathrm{E}-03$ & -20 & $5.67 \mathrm{E}-03$ & -18 & $5.15 \mathrm{E}-03$ & $6.20 \mathrm{E}-03$ \\
\hline${ }^{99 \mathrm{~m}} \mathrm{Tc}$ Technetium tetrofosmin, resting subject & $8.0 \mathrm{E}-03$ & 5.92E-03 & -26 & $6.15 \mathrm{E}-03$ & -23 & 5.57E-03 & $6.72 \mathrm{E}-03$ \\
\hline $\begin{array}{l}{ }^{99 m} \mathrm{Tc} \text { Technetium labelled white blood cells } \\
\text { (leukocytes) }\end{array}$ & $1.1 \mathrm{E}-02$ & $1.28 \mathrm{E}-02$ & 16 & $1.02 \mathrm{E}-02$ & -7 & 9.24E-03 & $1.12 \mathrm{E}-02$ \\
\hline${ }^{111}$ In Indium labelled HIG & 1.7E-01 & $2.23 \mathrm{E}-01$ & 31 & $2.15 \mathrm{E}-01$ & 26 & $1.99 \mathrm{E}-01$ & 2.31E-01 \\
\hline $\begin{array}{l}{ }^{111} \text { In Indium labelled monoclonal antibodies, } \\
\text { intact antibody }\end{array}$ & $3.3 \mathrm{E}-01$ & $2.88 \mathrm{E}-01$ & -13 & $2.74 \mathrm{E}-01$ & -17 & 2.49E-01 & 2.99E-01 \\
\hline${ }^{111}$ In Indium octreotide & $5.4 \mathrm{E}-02$ & $6.74 \mathrm{E}-02$ & 25 & $5.93 \mathrm{E}-02$ & 10 & $5.34 \mathrm{E}-02$ & $6.51 \mathrm{E}-02$ \\
\hline${ }^{123}$ I lodide, thyroid uptake $35 \%$ & $2.2 \mathrm{E}-01$ & $2.59 \mathrm{E}-01$ & 18 & $2.13 \mathrm{E}-01$ & -3 & $1.95 \mathrm{E}-01$ & 2.30E-01 \\
\hline${ }^{123}$ I lodine BMIPP & $1.6 \mathrm{E}-02$ & $1.70 \mathrm{E}-02$ & 6 & $1.71 \mathrm{E}-02$ & 7 & $1.56 \mathrm{E}-02$ & 1.87E-02 \\
\hline${ }^{123}$ | lodine IPPA & $1.6 \mathrm{E}-02$ & $1.72 \mathrm{E}-02$ & 7 & $1.72 \mathrm{E}-02$ & 8 & $1.56 \mathrm{E}-02$ & 1.87E-02 \\
\hline${ }^{123}$ I lodine labelled brain receptor substances & $5.0 \mathrm{E}-02$ & $3.60 \mathrm{E}-02$ & -28 & $3.65 \mathrm{E}-02$ & -27 & $3.30 \mathrm{E}-02$ & 4.00E-02 \\
\hline${ }^{123}$ I lodine Hippuran, normal renal function & $1.2 \mathrm{E}-02$ & 8.88E-03 & -26 & 7.06E-03 & -41 & $5.98 \mathrm{E}-03$ & $8.15 \mathrm{E}-03$ \\
\hline \multirow[t]{2}{*}{${ }^{123}$ I lodine MIBG } & $1.3 \mathrm{E}-02$ & $1.67 \mathrm{E}-02$ & 28 & 1.67E-02 & 28 & $1.51 \mathrm{E}-02$ & $1.82 \mathrm{E}-02$ \\
\hline & $2.9 \mathrm{E}-02$ & 3.29E-02 & 13 & 2.94E-02 & 1 & $2.68 \mathrm{E}-02$ & $3.21 \mathrm{E}-02$ \\
\hline
\end{tabular}


Table 1 Effective dose from the 55 radiopharmaceuticals in ICRP publication 106, determined using three different methods (Continued)

\begin{tabular}{|c|c|c|c|c|c|c|c|}
\hline \multicolumn{8}{|l|}{$\begin{array}{l}123 \text { l lodine labelled monoclonal antibodies, } \\
\text { intact antibody }\end{array}$} \\
\hline${ }^{124}$ I lodide, thyroid uptake $35 \%$ & $1.5 \mathrm{E}+01$ & $1.41 E+01$ & -6 & $1.15 E+01$ & -23 & $1.05 E+01$ & $1.25 \mathrm{E}+01$ \\
\hline${ }^{125}$ I lodide, thyroid uptake $35 \%$ & $1.4 \mathrm{E}+01$ & $1.85 \mathrm{E}+01$ & 32 & $1.50 \mathrm{E}+01$ & 7 & $1.38 \mathrm{E}+01$ & $1.62 E+01$ \\
\hline${ }^{131}$ I lodide, thyroid uptake $35 \%$ & $2.4 \mathrm{E}+01$ & $2.68 \mathrm{E}+01$ & 11 & $2.15 E+01$ & -10 & $1.98 \mathrm{E}+01$ & $2.33 \mathrm{E}+01$ \\
\hline${ }^{131}$ | lodine, Hippuran, normal renal function & $5.2 \mathrm{E}-02$ & 1.89E-02 & -64 & $1.53 \mathrm{E}-02$ & -71 & 1.29E-02 & 1.78E-02 \\
\hline $\begin{array}{l}{ }^{131} \text { I lodine, labelled monoclonal antibodies, } \\
\text { intact antibody }\end{array}$ & 4.7E-01 & 4.40E-01 & -6 & 3.59E-01 & -24 & $3.26 \mathrm{E}-01$ & 3.94E-01 \\
\hline${ }^{131} \mid$ lodine NP59 & $1.8 \mathrm{E}+00$ & $2.02 \mathrm{E}+00$ & 12 & $1.74 \mathrm{E}+00$ & -3 & $1.60 \mathrm{E}+00$ & $1.89 \mathrm{E}+00$ \\
\hline${ }^{201} \mathrm{TI}$ Thallium ion & $1.4 \mathrm{E}-01$ & $1.27 \mathrm{E}-01$ & -10 & 1.02E-01 & -27 & $9.90 \mathrm{E}-02$ & $1.05 \mathrm{E}-01$ \\
\hline
\end{tabular}


8. "Using the new estimations, the collective effective dose is estimated at 292 manSv, i.e. 13 \% lower value than earlier estimated." Should be "Using the new estimations, the collective effective dose is estimated at 295 manSv, i.e. $12 \%$ lower value than earlier estimated."

In the Conclusions there are two sentences that should be changed

1. "For 268 radiopharmaceuticals out of 338, the new calculations show lower effective dose values than previous estimates." Should be "For 212 radiopharmaceuticals out of 338, the new calculations show lower effective dose values than previous estimates."

2. "For 68 radiopharmaceuticals, the new calculations results in an increased value of the estimated effective dose." Should be "For 120 radiopharmaceuticals, the new calculations results in an increased value of the estimated effective dose."

Figure 1 should be changed to (only the figure not the text)

Almost all numbers have been changed in Table 1 and a new corrected Table 1 is presented below (table text to Table 1 does not need to be changed).

A new Supplemental file is given in a separate file named "Additional file 1: Table S1".

\section{Additional file 1}

Additional file 1: Table S1. Effective dose from all the radiopharmaceuticals published by the ICRP, determined using three different methods. $\left(E / A_{0}\right) 1$ is the previously published effective dose per unit administered activity $\left(E / A_{0}\right)$ by ICRP, $\left(E / A_{0}\right) 2$ is $\left(E / A_{0}\right)$ dose calculated with the new phantoms and old tissue weighting factors while $\left(E / A_{0}\right) 3$ is with the new phantoms and new weighting factors. $\left.\left.\left(E / A_{0}\right) 2-\left(E / A_{0}\right) 1\right)\right) /\left(E / A_{0}\right) 1$ and $\left(\left(E / A_{0}\right) 3-\left(E / A_{0}\right) 1\right) /$ $\left(E / A_{0}\right) 1$ is the difference in \% of the new values compared to the old. $\left(E / A_{0}\right) 3$ male and $\left(E / A_{0}\right) 3$ are the effective dose estimations generated from the equivalent dose of each gender separately using the new phantoms and new weighting factors. (DOCX $65 \mathrm{~kb}$ )

Published online: 30 September 2015

Submit your manuscript to a SpringerOpen ${ }^{\circ}$ journal and benefit from:

- Convenient online submission

- Rigorous peer review

- Immediate publication on acceptance

- Open access: articles freely available online

- High visibility within the field

- Retaining the copyright to your article

Submit your next manuscript at $>$ springeropen.com 